\title{
Comparison of the efficacy of using paclitaxel-eluting balloon and plain balloon angioplasty for arteriovenous fistula in hemodialysis patients
}

\author{
Alireza Rai $^{1(0)}$, Mohammadreza Sobhiyeh ${ }^{2, *(1)}$
}

${ }^{1}$ Department of cardiology, Imam ali hospital, kermanshah university of medical sciences, kermanshah, Iran

${ }^{2}$ Department of vascular Endovascular surgery, Imam reza hospital, kermanshah university of medical sciences, kermanshah, Iran

\section{Correspondence}

Mohammadreza Sobhiyeh, Department of vascular Endovascular surgery ,Imam reza hospital, kermanshah university of medical sciences, kermanshah, Iran

Email: mreza.sobhiyeh@yahoo.com

\section{History}

- Received: Mar 07, 2019

- Accepted: Apr 30, 2019

- Published: May 30, 2019

DOI :

https://doi.org/10.15419/bmrat.v6i5.541

\section{Check for updates}

\section{Copyright}

(c) Biomedpress. This is an openaccess article distributed under the terms of the Creative Commons Attribution 4.0 International license.

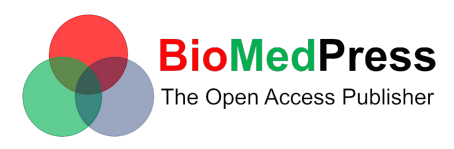

\begin{abstract}
Introduction: Arteriovanous (AV) access failure is one of the main problems in patients with end stage renal disease (ESRD), who receive hemodialysis. Balloon angioplasty is a favorable method for managing vascular access failure. The purpose of this study was to compare the six-month efficacy of paclitaxel-eluting balloon and plain balloon angioplasty in failed AV access cases among hemodialysis patients. Methods: In this quasi-experimental study (http://en.irct.ir/trial/35333), 50 hemodialysis patients with failure of AV access (stenosis > 50\%), who were candidates for angioplasty, were included. They were divided to receive either paclitaxel-eluting balloon (25 patients) or plain balloon (25 patients) angioplasty. Patients were followed up for six months with color Doppler ultrasonography and clinical examination for the hemodynamic success rate of angioplasty. Results: After six months, 19 patients (76\%) in paclitaxel-eluting balloon angioplasty group achieved hemodynamic success, which was significantly higher than plain balloon angioplasty group (13 patients, 52\%) ( $P=0.012$ ). Age, gender, diabetes mellitus, hypertension, and location of AVF (snuff box, forearm, and antecubital fossa) did not associate with hemodynamic success rate in any group. Conclusion: The use of angioplasty with paclitaxel-eluting balloon was superior to plain balloon angioplasty for failed AV access cases in hemodialysis patients. It is recommended to use paclitaxeleluting balloon angioplasty in patients with failure of AV access and requirement for balloon angioplasty.

Key words: Arteriovenous access, Hemodialysis, angioplasty, paclitaxel-eluting balloon
\end{abstract}

\section{INTRODUCTION}

Arteriovenous (AV) access is one of the best and least complicated vascular access methods in hemodialysis patients. In comparison to other vascular access methods (central vein access), AV access has the lowest rate of complications such as thrombosis and infection ${ }^{1}$. Therefore, AV access is usually considered the first choice for long-term hemodialysis patients ${ }^{2}$. The complications of vascular access are the main cause of failure, which increases the costs for patients in the final stages of chronic renal failure. Also, poor functioning $\mathrm{AV}$ access is the most common cause of intervention and re-admission of the patients ${ }^{3}$.

Stenosis in the arterial-venous pathway is a cause of dysfunction of the AV accesses. The causes of this complication include cell proliferation, secretion of cytokines by endothelial cells, smooth muscle, and macrophages. Cytokine secretion leads to cellular activity and vascular hyperplasia ${ }^{4}$. In addition to the location and type of fistula, several factors such as age, underlying disease, peripheral vascular disease, the onset and history of dialysis and central catheter can affect the function of AV access.

Angioplasty is an effective treatment for arterial dysfunction such as stenosis in the artery, vein or anastomosis ${ }^{5}$. Using balloon angioplasty, stenosis of the artery and vein is resolved. One of the treatments with minimally invasive angiography is to restore the inserted fistula to dialysis patients. It is improved by the insertion of a simple stent or drug-coated stent, or endothelial anti-proliferation to restore the arterial flow ${ }^{6}$.

The proposed mechanisms for re-stenosis in the AV accesses include uremia, oxidative stress, and inflammation, which result in endothelial dysfunction in patients with ESRD (end stage renal disease), uremia, vasodilatation and increased stress that cause negative remodeling in the blood vessels. Stenosis of the $\mathrm{AV}$ accesses affects $60 \%$ of venous access, $20 \%$ of AV anastomosis and $20 \%$ of central venous catheters ${ }^{7,8}$. As most access implants are fibrotic, the dilatation is challenging with conventional balloons. To date, a number of new techniques, including high pressure balloons or cutting balloons, are being developed to 
improve to achieve a normal diameter in 35 to 40 percent of cases. But all these therapeutic strategies are accompanied by some rates of re-stenosis ${ }^{9}$.

The role of paclitaxel-eluting balloons as an antirestenosis strategy to reduce intimal hyperplasia using anti-proliferative drug (paclitaxel) is promising ${ }^{10}$. Several studies have been conducted regarding drugcoated balloons that showed the benefits of using these paclitaxel-eluting balloons ${ }^{8,11,12}$.

The objective of this study was to compare the efficacy of angioplasty with paclitaxel-eluting balloons and plain balloons for failed AV access in ESRD patients undergoing hemodialysis. The results of this study can provide valuable information on the preferred method for managing patency of AV access in patients with ESRD.

\section{METHODS}

\section{Study design and population}

This was a quasi-experimental study. The study population included patients with ESRD, who were referred to our educational hospital in 2017 due to the failure of $\mathrm{AV}$ access and were appointed for balloon angioplasty.

Evaluation of AVF failure was performed with AVF venography or ultrasound, which showed at least $50 \%$ stenosis at the proximal part of the vein without aneurysm. Exclusion criteria included patients with use of anticoagulant drugs, immune dysfunction or those who were taking immunosuppressive drugs, those who could not be physically operated during surgery, the inability to follow the patients, discontinuation of hemodialysis, and those who did not consent for angioplasty.

\section{Data collection}

Upon enrollment and before the operation, physical examination and documentation of vital signs including blood pressure (BP) measurement were performed. In addition, medical records were reviewed, and past medical history and drug history were taken from all patients. Hypertension was defined when systolic BP $\geq 140 \mathrm{mmHg}$ and/or diastolic $\mathrm{BP} \geq 90 \mathrm{mmHg}$, with previous history of high systolic/diastolic BP and anti-hypertensive medications. Diabetes mellitus (DM) was defined when fasting blood glucose $\geq 126 \mathrm{mg} / \mathrm{dL}$ and HbAlC level $\geq$ $6.5 \%$ or with previous documented diagnosis of DM and oral anti-diabetics or insulin intake. In addition, the etiology of renal failure was collected from medical records.

The collected variables included demographic data, evidence of DM determined by fasting blood glucose and $\mathrm{HbA1C}$, BP measurement, AVF location, and the follow-up success rates.

\section{Procedures}

All procedures were performed by a single vascular surgeon using the same method. The procedure was performed under regional anesthesia by micropuncture technique. Using ultrasound, venous puncture was done in a retrograde fashion and digital venography was performed. Then, the location of the lesion, and its length and diameter were determined. After that, 2,500 units of heparin was injected. In the plain balloon group, high pressure balloons (CONQUEST PTA Dilatation, Bard Peripheral Vascular, USA) were used. The diameter of the balloon was equal to the proximal part of the vein and its length was equal to the length of the stenosis. In paclitaxel-eluting balloon group, high pressure balloons (Lutonix ${ }^{\circ}$, Bard Peripheral Vascular, USA) were used. According to the instructions of the manufacturer, the length of the balloon was $1 \mathrm{~mm}$ longer than the stenosis, and its diameter was $1 \mathrm{~mm}$ larger than the diameter of the stenosis. The balloons were inflated for 3 minutes.

\section{Follow-up}

Patients were examined at three- and six-month intervals for the presence or absence of thrill and bruits. In addition, color Doppler sonography was performed to determine AVF blood. Success was defined when residual stenosis with plain balloon was less than $30 \%$ and/or as hemodynamic success. If partial dilation of proximal venous in the extremity was confirmed, the vascular access was considered as patent and hemodialysis was continued.

\section{Statistical analyses}

To report variables, descriptive statistics including mean and standard deviation (SD) were used. For comparing the qualitative variables between the two groups, Chi-square test was used. In order to compare the quantitative variables, $\mathrm{t}$-test or Mann-Whitney $\mathrm{U}$ test was used considering the normal distribution of continuous data. To compare the patency of AVF and hemodynamic success at 3 and 6 months after angioplasty, repeated measured ANOVA (analysis of variance) was used. The analyses were performed using SPSS software (Ver. 20.0).

\section{Ethics}

The study protocol was approved by the Ethics Committee of our university (IR.KUMS.REC.1397.518). The study conforms to the Declaration of Helsinki. 


\section{RESULTS}

\section{Baseline variables}

The total of 50 patients ( 24 male and 26 female) with mean $( \pm S D)$ age of $50.31( \pm 3.96)$ years (ranged from 32 to 70 ) were included. They were divided into two groups: one group underwent angioplasty with paclitaxel-eluting balloons (25 patients) and the other group received angioplasty with plain balloons (25 cases).

Table 1 presents gender distribution, diabetes mellitus frequency and hypertension frequency in the studied groups. As observed, there was no significant difference between the groups regarding gender, age, frequency of diabetes mellitus and hypertension. Table 2 presents causes of ESRD in each group. Regarding to the location of AV accesses, snuff box was the most common location seen in paclitaxel-eluting balloons (13 cases, $52 \%$ ) and plain balloon (15 cases, $60 \%$ ) groups, followed by forearm (7 cases $(28 \%)$ vs. 6 cases $(24 \%))$ and antecubital fossa (5 cases $(20 \%) v s$. 4 cases $(16 \%))$.

Hemodynamic success rate at 3- and 6month follow-up

At three months, hemodynamic success rate was $84 \%$ (21 patients) in paclitaxel-eluting balloon group and $88 \%$ (22 cases) in plain balloon angioplasty group. After six months, 19 patients (76\%) in paclitaxeleluting balloon angioplasty group achieved hemodynamic success, which was significantly higher than plain balloon angioplasty group (13 patients, 52\%) (P $=0.012$ ).

Hemodynamic success rate based on age, gender, comorbidities, and AV access location

Table 3presents hemodynamic success rate at 6 months in paclitaxel-eluting balloon angioplasty group based on age, gender, and comorbidities. No difference was found between patent and failed $\mathrm{AV}$ accesses regarding mean age, gender distribution, comorbidities and location of AV access. Table 4presents patency of AV accesses based on the mentioned variables in plain balloon angioplasty group. The mentioned variables had no association with hemodynamic success rate in plain balloon angioplasty group.

\section{DISCUSSION}

The use of AV access has led to significant improvements in the management of patients requiring hemodialysis. With regard to the limitation of the location of AV accesses in each patient, and to avoid unnecessary costs and frequent visits to the hospital, the effectiveness of $\mathrm{AV}$ accesses and recognizing the factors affecting their patency is important. However, vascular access failure is one of the challenging conditions regarding AV accesses. Hence, studies are carried out to discover the more effective ways, including plain balloon percutaneous angioplasty, to improve the function of failed AV accesses. However, there is concern regarding long-term efficacy of plain balloons ${ }^{12}$. One of the methods that is studied recently is the use of paclitaxel-eluting balloons instead of plain balloons. It is suggested that paclitaxel can reduce intimal hyperplasia, as a major factor for stenosis, which can be more successful in long-term over plain balloons ${ }^{11}$. Based on the obtained findings, paclitaxeleluting balloon angioplasty was superior to plain balloon angioplasty regarding the patency of AV access after six months in hemodialysis patients with failed $\mathrm{AV}$ access.

In a similar study ${ }^{11}$, the authors investigated paclitaxel-eluting balloon angioplasty vs. plain balloon angioplasty among 40 patients with stenosis of AV accesses. They reported that patency at 1 year was significantly higher in paclitaxel-eluting balloon group (35\%) compared to just 5\% in plain balloon group. These results are in agreement with our findings indicating the advantage of paclitaxeleluting balloons, albeit we followed the patients for six months. We observed that at 3 months, no difference was seen between the groups regarding hemodynamic success rate. The previous study ${ }^{11}$ did not report results in short term. It is possible that the best outcomes regarding paclitaxel-eluting balloons are documented after at least six months of angioplasty.

In a separate study including 40 patients $^{8}$, the authors investigated the 6-month outcome of paclitaxelcoated balloon angioplasty for failed AV access. They noted that in comparison to plain balloon angioplasty, the patients for whom paclitaxel-coated balloons were used had higher rate of patency (70\%) compared to the other group (25\%) after six months. On the other hand, procedural success was similar in both groups (100\%).

The maintenance of patency of AV access, as the most popular vascular access in hemodialysis patients, is of great importance. In the case of failing AV access and stenosis, interventional procedures are usually implemented ${ }^{8}$. The use of paclitaxel-eluting balloons is a promising approach based on the findings obtained 
Table 1: Comparison of the studied variables between two groups of patients with failed arteriovenous fistula (AVF) who underwent angioplasty

\begin{tabular}{llll}
\hline & $\begin{array}{l}\text { Paclitaxel-eluting balloon }(\mathbf{N}= \\
\mathbf{2 5})\end{array}$ & Plain balloon $(\mathbf{N}=\mathbf{2 5})$ & P-value \\
Gender, male & $11(44 \%)$ & $13(52 \%)$ & 0.84 \\
Age & $49.24( \pm 4.03)$ & $48( \pm 17.3)$ & 0.64 \\
Diabetes mellitus & $11(44 \%)$ & $17(68 \%)$ & 0.81 \\
Hypertension & $15(60 \%)$ & $12(48 \%)$ & 0.65 \\
\hline
\end{tabular}

Table 2: Causes of end-stage renal disease (ESRD) in two groups of patients with failed arteriovenous fistula (AVF) who underwent balloon angioplasty

\begin{tabular}{lcc}
\hline & $\begin{array}{r}\text { Paclitaxel-eluting balloon } \\
(\mathbf{N}=\mathbf{2 5})\end{array}$ & $\begin{array}{r}\text { Plain balloon } \\
\mathbf{( N = 2 5 )}\end{array}$ \\
Hypertension & $12(48 \%)$ & $8(32 \%)$ \\
Diabetes mellitus & $9(36 \%)$ & $10(40 \%)$ \\
Polycystic kidney & $1(4 \%)$ & $2(8 \%)$ \\
Diabetes mellitus and hypertension & $3(12 \%)$ & $2(8 \%)$ \\
Glumerulonephritis & 0 & $2(8 \%)$ \\
Nephrolithiasis & 0 & $1(4 \%)$ \\
\hline
\end{tabular}

Table 3: Comparison of hemodynamic success rate of Paclitaxel-eluting balloon angioplasty for failed arteriovenous fistula (AVF) based on age, gender, and comorbidities

\begin{tabular}{|c|c|c|c|c|}
\hline & & $\begin{array}{l}\text { Patent AVF } \\
(\mathrm{N}=19)\end{array}$ & $\begin{array}{l}\text { Failed AVF } \\
(\mathrm{N}=6)\end{array}$ & P-value \\
\hline Age, year & & $48.13( \pm 2.5)$ & $50.24( \pm 6.1)$ & 0.31 \\
\hline Gender, male & & $5(20 \%)$ & $6(24 \%)$ & 0.51 \\
\hline Diabetes mellitus & & $4(16 \%)$ & $7(28 \%)$ & 0.09 \\
\hline Hypertension & & $4(16 \%)$ & $7(28 \%)$ & 0.91 \\
\hline \multirow[t]{3}{*}{ Location of AVF } & Snuff box & $6(50 \%)$ & $6(50 \%)$ & 0.7 \\
\hline & Forearm & $3(12 \%)$ & $4(16 \%)$ & \\
\hline & $\begin{array}{l}\text { Antecubital } \\
\text { fossa }\end{array}$ & $2(8 \%)$ & $3(12 \%)$ & \\
\hline
\end{tabular}

\begin{tabular}{|c|c|c|c|c|}
\hline & & Patent AVF $(\mathrm{N}=19)$ & Failed AVF $(\mathrm{N}=6)$ & $P$ value \\
\hline Age, year & & $46.87(7.2)$ & $52.78(3.4)$ & 0.41 \\
\hline Gender, male & & $8(32 \%)$ & $7(28 \%)$ & 0.71 \\
\hline Diabetes mellitus & & $5(20 \%)$ & $12(48 \%)$ & 0.06 \\
\hline Hypertension & & $5(20 \%)$ & $7(28 \%)$ & 0.86 \\
\hline \multirow[t]{3}{*}{ Location of AVF } & Snuff box & $10(36 \%)$ & $5(20 \%)$ & \\
\hline & Forearm & $3(12 \%)$ & $3(12 \%)$ & \\
\hline & Antecubital fossa & $2(8 \%)$ & $2(8 \%)$ & \\
\hline
\end{tabular}


here and previous reports. This approach is more advantageous than other options, such as a second $\mathrm{AV}$ access, which can be associated with infectious complications $^{2}$. As intimal hyperplasia is a known cause of stenosis and dysfunction of AV accesses, the use of paclitaxel-eluting balloons that have anti-proliferative effects can prohibit vascular access failure, especially after 6 months of angioplasty.

We faced some limitations in performing this study. First, this was a quasi-experimental study and thus, randomization was not performed. However, no significant difference was seen based on baseline variables between the two studied groups. Further studies with randomization are suggested. In addition, we followed patients for six months. Longer follow-ups are also suggested for better characterization of patency of paclitaxel-eluting balloons in long term.

\section{CONCLUSIONS}

The use of angioplasty with paclitaxel-eluting balloon was superior to plain balloon angioplasty for failed AV access in hemodialysis patients. It is recommended to use paclitaxel-eluting balloon angioplasty in patients with failure of $\mathrm{AV}$ access and requirement for angioplasty with balloons.

\section{ABBREVIATIONS}

AVF: Arteriovanous fistula

ESRD: end-stage renal disease

BP: blood pressure

DM: diabetes mellitus

\section{COMPETING INTERESTS}

The authors declare that there is no conflict of interest regarding the contents of this article.

\section{AUTHORS' CONTRIBUTIONS}

AR proposed the concept of the research. SA performed data gathering, follow up of the patients, and statistical analyses. MS drafted the article and supervised the research.

\section{ACKNOWLEDGMENTS}

This article is part of an M.D thesis by Amiri S (97805), approved by the Research Deputy of Kermanshah University of Medical Sciences. The authors wish to thank all staffs of the Peripheral intervention ward of Imam Reza Hospital for their cooperation in this study. We appreciate the clinical research development center of imam Reza Hospital Kermanshah University of medical Sciences for their wise advice.

\section{REFERENCES}

1. Stolic R. Most important chronic complications of arteriovenous fistulas for hemodialysis. Med Princ Pract. 2013;22(3):220-8. 23128647. Available from: 10.1159/ 000343669 .

2. Al-Balas A, Lee T, Young CJ, Allon M. Choice of a second vascular access in hemodialysis patients whose initial arteriovenous fistula failed to mature. J Vasc Surg. 2018;68(6). 29937290. Available from: 10.1016/j.jvs.2018.03.419.

3. Ortega T, Ortega F, Diaz-Corte C, Rebollo P, Baltar JM, AlvarezGrande J. The timely construction of arteriovenous fistulae: a key to reducing morbidity and mortality and to improving cost management. Nephrol Dial Transplant. 2005;20(3):598603. 15647308. Available from: 10.1093/ndt/gfh644.

4. Roy-Chaudhury P, Kelly BS, Miller MA, Reaves A, Armstrong J, Nanayakkara $\mathrm{N}$, et al. Venous neointimal hyperplasia in polytetrafluoroethylene dialysis grafts. Kidney Int. 2001;59(6):2325-34. 11380837. Available from: 10.1046/j. 1523-1755.2001.00750.x.

5. Duque JC, Tabbara M, Martinez L, Cardona J, Vazquez-Padron RI, Salman LH. Dialysis Arteriovenous Fistula Failure and Angioplasty: Intimal Hyperplasia and Other Causes of Access Failure. Am J Kidney Dis. 2017;69(1):147-51. 28084215. Available from: 10.1053/j.ajkd.2016.08.025.

6. Peden EK, Leeser DB, Dixon BS, El-Khatib MT, Roy-Chaudhury $\mathrm{P}$, Lawson JH. A Multi-center, Dose-escalation Study of Human type I Pancreatic Elastase (PRT-201) Administered after Arteriovenous Fistula Creation. The Journal of Vascular Access. 2013;14(2):143-51. 23172172. Available from: 10.5301/ jva. 5000125 .

7. Drew DA, Lok CE, Cohen JT, Wagner M, Tangri N, Weiner $\mathrm{DE}$. Vascular access choice in incident hemodialysis patients: a decision analysis. J Am Soc Nephrol. 2015;26(1):183-91. 25063436. Available from: 10.1681/asn.2013111236.

8. Katsanos K, Karnabatidis D, Kitrou P, Spiliopoulos S, Christeas $\mathrm{N}$, Siablis D. Paclitaxel-coated balloon angioplasty vs. plain balloon dilation for the treatment of failing dialysis access: 6month interim results from a prospective randomized controlled trial. J Endovasc Ther. 2012;19(2):263-72. 22545894. Available from: 10.1583/11-3690.1.

9. Agarwal SK, Nadkarni GN, Yacoub R, Patel AA, Jenkins JS, Collins TJ, et al. Comparison of Cutting Balloon Angioplasty and Percutaneous Balloon Angioplasty of Arteriovenous Fistula Stenosis: A Meta-Analysis and Systematic Review of Randomized Clinical Trials. J Interv Cardiol. 2015;28(3):288-95. 25989717. Available from: 10.1111/joic.12202.

10. Terry CM, Li L, Li H, Zhuplatov I, Blumenthal DK, Kim SE, et al. In vivo evaluation of the delivery and efficacy of a sirolimusladen polymer gel for inhibition of hyperplasia in a porcine model of arteriovenous hemodialysis graft stenosis. J Control Release. 2012;160(3):459-67. 22465391. Available from: 10. 1016/j.jconrel.2012.03.011.

11. Kitrou PM, Katsanos K, Spiliopoulos S, Karnabatidis D, Siablis D. Drug-eluting versus plain balloon angioplasty for the treatment of failing dialysis access: final results and costeffectiveness analysis from a prospective randomized controlled trial (NCT01174472). Eur J Radiol. 2015;84(3):418-23. 25575743. Available from: 10.1016/j.ejrad.2014.11.037.

12. LuJ, Breznik S, Dinevski D, Ekart R, Rupreht M. Endovascular Treatment of Haemodialysis Arteriovenous Fistula with DrugCoated Balloon Angioplasty: A Single-Centre Study. Cardiovasc Intervent Radiol. 2018;41(6):882-9. 29582126. Available from: $10.1007 / \mathrm{s} 00270-018-1942-\mathrm{z}$. 